PRAXIS

ecucativa

Universidad Nacional de La Pampa

Facultad de Ciencias Humanas

Instituto de Ciencias de la Educación

para la investigación interdisciplinaria

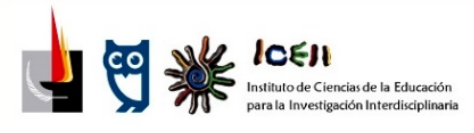

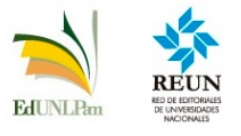

ISSN 2313-934X

SANTA ROSA, LA PAMPA, ARGENTINA

Correo electrónico: iceii@humanas.unlpam.edu.ar

Disponible en https://cerac.unlpam.edu.ar/index.php/praxis

La "discusión" en las aulas. Un estudio sobre las experiencias de formación ciudadana en escuelas secundarias de Córdoba, Argentina. Artículo de Andrés E. Hernández. Praxis educativa, Vol. 25, No 3 septiembre - diciembre 2021. E - ISSN 2313-934X. pp. 1-17. https://dx.doi.org/10.19137/praxiseducativa-2021-250313

Esta obra se publica baja Licencia Creative Commons 4.0 Internacional

CC BY- NC-SA Atribución, No Comercial, Compartir igual

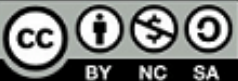

\title{
La "discusión" en las aulas. Un estudio sobre las experiencias de formación ciudadana en escuelas secundarias de Córdoba, Argentina
}

The "discussion" in the classrooms. A study about citizen training experiences in secondary schools in Córdoba, Argentina

A discussão nas aluas. Um estudo sobre as experiências de formação cidadã nas escolas de ensino médio de Córdoba, Argentina

\section{Andrés E. Hernández}

Instituto Académico Pedagógico de Ciencias Sociales de la Universidad Nacional de Villa María IAPCS-CONICET Argentina

undher@gmail.com

ORCID: 0000-0001-7736-5241

Recibido: 2021-02-02 | Revisado: 2021-06-09 | Aceptado: 2021-07-20 


\section{Resumen}

En este artículo, se abordan las experiencias de formación ciudadana de jóvenes que asisten a escuelas secundarias en Argentina. Luego de revisar y sistematizar parte de los estudios que se han enfocado en cuestiones vinculadas a la educación ciudadana, se fundamenta acerca de la relevancia de incorporar el análisis de las "discusiones" de temas controvertidos que tienen lugar cotidianamente en las escuelas. En este sentido, se intenta mostrar cómo el enfoque etnográfico, que implica la presencia prolongada del investigador en campo, permite identificar y seguir de cerca los modos en que se estructuran las relaciones entre estudiantes, docentes y padres. De este modo, resulta posible distinguir y analizar una serie de procesos en los cuales se expresan diferentes y desiguales modos de construcción de ciudadanía.

Palabras clave: educación ciudadana; participación política; escuela secundaria; jóvenes; relaciones intergeneracionales.

\section{Abstract}

This article offers an approach to the citizenship training experiences of young people who attend secondary schools in Argentina. After reviewing and systematizing part of the studies that have focused on issues related to citizenship education, it is based on the relevance of incorporating the analysis of the "discussions" of controversial issues that take place daily in schools. In this sense, an attempt is made to show how the ethnographic approach, which implies the prolonged presence of the researcher in the field, makes it possible to identify and closely monitor the ways in which the relationships between students, teachers and parents are structured. In this way, it is possible to distinguish and analyze a series of processes in which different and unequal ways of constructing citizenship are expressed.

Keywords: citizenship education; political participation; secondary school; youths; intergenerational relationships.

\section{Resumo}

Neste artigo, abordam-se as experiências de formação cidadã de jovens que assistem a escolas de ensino médio na Argentina. Após verificar e sistematizar parte dos estudos que focaram em questões relacionadas à educação cidadã, fundamenta-se sobre a importância de incorporar a análise das "discussões" de temas controvertidos que diariamente ocorrem nas escolas. Neste sentido, tenta-se mostrar como o foco etnográfico, que envolve a presença prolongada do investigador no campo, permite identificar e acompanhar de perto as maneiras em que se estruturam as relações entre estudantes, professores e pais. Desta forma, é possível diferenciar e analisar uma série de processos nos quais se expressam diferentes e desiguais modos de construção da cidadania.

Palavras-chave: educação cidadã; participação política; ensino médio; jovens; relações inter geracionais. 


\section{Introducción}

El presente trabajo se propone profundizar el análisis de aquellas prácticas y representaciones que tienen lugar en escenarios escolares y que resultan inteligibles en la medida en que se pone el foco en la dimensión política de las experiencias educativas. Para ello, se ponen en consideración una serie de contrastes y diferencias que resultan visibles solamente a partir de la presencia prolongada del investigador en campo y que permiten dar cuenta de la diversidad de experiencias vinculadas a la formación ciudadana de jóvenes que cursan sus estudios secundarios. El artículo está organizado en tres partes. En la primera, se propone una breve sistematización de los principales debates en torno a la educación ciudadana en Argentina. Allí, se repasan algunos de los hallazgos e hipótesis que han sostenido las distintas líneas de investigación que conforman este vasto campo de estudios, para luego fundamentar la necesidad de incorporar el análisis de la "discusión" de temas controvertidos en las escuelas. En el segundo apartado, se detallan algunas consideraciones acerca de la estrategia metodológica asumida, la cual se corresponde con un tipo de enfoque y de trabajo de campo que resulta especialmente útil a los fines de identificar y documentar las texturas y las tramas que configuran el cotidiano de las experiencias escolares. Finalmente, en la tercera parte, se propone un análisis y sistematización de algunos de los datos recogidos y coproducidos a lo largo del trabajo de campo realizado en tres escuelas secundarias de la ciudad de Córdoba, Argentina. Allí, se intentará mostrar cierta clase de diferencias y desigualdades que se expresan en las experiencias escolares y que están implicadas en la configuración de un modo particular de formación ciudadana. La "discusión", como veremos, ocupa un lugar fundamental en relación con las dinámicas y las relaciones que las "clases de ciudadanía" habilitan. Seguir los rastros de estos intercambios, entre distintas posiciones y puntos de vista acerca de las más variadas cuestiones políticas, permite dar cuenta de los modos en que se van estructurando los diversos procesos de formación ciudadana que las escuelas, hoy, hacen posibles.

\section{Antecedentes}

Los estudios sobre educación ciudadana gozan de una amplia tradición en Argentina. Algunos trabajos, como los de Isabelino Siede y Gustavo Schujman (Siede, 2007; Siede y Schujman, 2007; Schujman, 2008; Siede et al., 2015), se han enfocado en la enseñanza y el currículum, mostrando las transformaciones históricas, así como las tensiones que se han manifestado a lo largo del siglo XX en el plano de la función política desempeñada por la escuela. De este modo, han dado cuenta de los distintos modos en que el sistema educativo ha resuelto aquel dilema acerca de si la escuela debiera ofrecer una educación en valores, o bien, una educación ciudadana, lo cual se corresponde, de algún modo, con el debate entre moral y política. En este sentido, tal como destaca Alucín (2018), la reapertura democrática de los años 80 representó el declive de la educación moral de la última dictadura militar y su reemplazo por una "Educación cívica", que se proponía formar un ciudadano moderado, tolerante y participativo, lo que implicó la apelación a una concepción consensual de política (2018, p. 66). No obstante ello, durante los 90, primó nuevamente la dimensión moral y jurídica por sobre la política e histórica (Alucín, 2018; Encabo, 
2008). Otros autores han destacado que el nuevo diagrama de contenidos de la asignatura Formación ética y ciudadana ${ }^{1}$-según los bloques persona, valores y normas- implicó, además, la introducción de saberes instrumentales de la filosofía práctica y la ética, junto con la profundización del abordaje de los Derechos Humanos (Ferreyra et al., 2014, p. 198).

En una comparación entre Argentina y Costa Rica, Suárez (2008) resalta el hecho de que los nuevos programas que siguieron a la sanción de la Ley Federal de Educación (N²4.195/1993) lograron, finalmente, expandir el foco de los derechos más allá de la tradicional concepción anclada en los límites del Estado-Nación. Así, los Derechos Humanos llegaron a convertirse en la pieza clave de las competencias que la escuela venía a desarrollar, relegando la cuestión de las responsabilidades de los ciudadanos, las ideas de nación y patriotismo y la enseñanza de la Constitución (Suárez, 2008). Una vez superado el escenario inmediato de postdictadura, el nuevo enfoque acerca de los derechos humanos como contenido trasversal habría sido determinante para provocar un salto hacia adelante en la renovación de los programas y los libros de texto, en los términos de una ciudadanía global.

Más recientemente, se ha podido observar un amplio consenso respecto a que los Núcleos de Aprendizaje Prioritarios, que siguieron a la sanción de la Ley de Educación Nacional (N026.206/2006), se tradujeron finalmente en asignaturas que incorporaron la diversidad y la participación ciudadana, otorgándole mayor centralidad a la cuestión de la identidad (Alucín, 2018; Ferreyra et al., 2014; Hernández, 2019). Esto confirma, de algún modo, la renovación y consolidación de un énfasis creciente, y relativamente estable, que pone a la cuestión política por encima de la dimensión ética o jurídica, lo que permitiría identificar una nueva etapa. La novedad estaría dada por el hecho de que ya no se trata solamente de la ampliación de los sentidos de la ciudadanía, ahora pensada en clave heterogénea y multicultural (Suárez, 2008), sino que, además, se tornan visibles las tensiones y los conflictos que tienen lugar en escenarios socialmente condicionados e históricamente determinados.

Ahora bien, cabe reconocer, por otra parte, que este proceso reciente de politización de la educación ciudadana, que puede ser constatado a nivel curricular sin mayores dificultades, no necesariamente se corresponde con la complejidad y la heterogeneidad que caracterizan a las experiencias, entramados y dinámicas institucional y localmente ancladas. En este sentido, otras líneas de investigación enfocadas en las prácticas y experiencias de los y las jóvenes han observado la relativa ampliación de aquellas concepciones más bien tradicionales acerca de la educación ciudadana, mostrando el alcance del viraje hacia un nuevo paradigma de la «ciudadanía democrática». De este modo, las escuelas secundarias han cobrado especial importancia en el marco de los renovados esfuerzos por comprender las transformaciones políticas que están teniendo lugar en un contexto que ha llegado a ser caracterizado como un nuevo ciclo de politización juvenil (Kriger, 2016). Allí confluyen, entre otros factores: i) la importancia de la

\footnotetext{
${ }^{1}$ Inspirada en los contenidos básicos comunes (CBC) según la resolución N³9/94, en el marco de la Ley Federal de Educación 24.195/93.
} 
«participación» y el involucramiento político en el marco de las políticas educativas y otras formas actuales de producción socioestatal de las juventudes (Vázquez, 2015), destacándose la referencia permanente al enfoque de derechos; y ii) la «democratización» de las escuelas, lo cual refiere no solo a la masificación creciente según la ampliación de los niveles de cobertura, sino además a la reconfiguración de las formas de sociabilidad y regulación de las conflictividades observadas al interior de las instituciones, lo cual torna visibles entre otras cosas el reemplazo -no exento de tensiones y contradicciones- de los regímenes disciplinares más tradicionales por nuevos paradigmas o sistemas centrados en la convivencia (Litichever, 2014; Litichever \& Núñez, 2005; Núñez \& Litichever, 2015).

En el marco de este segundo conjunto de investigaciones enfocadas en las prácticas y experiencias escolares juveniles, Núñez $(2014 ; 2019)$ ha llamado la atención acerca de las diferencias y desigualdades entre sectores sociales, que se expresarían también en las formas de construcción de ciudadanía. En este sentido, y a modo de hipótesis, sostiene que las experiencias educativas juveniles adquieren formas disímiles según las tradiciones políticas existentes en las instituciones, los grados de heterogeneidad/homogeneidad de la matrícula escolar y los repertorios de acción a los que apelan los y las estudiantes (Núñez, 2014, p. 49). Así, en la medida en que las escuelas gozan de mayor autonomía para definir sus propias regulaciones pueden observarse distintos efectos relativos al conocimiento o no de las normativas, como así también respecto de las formas de percepción de las injusticias experimentas por los estudiantes en las escuelas (Núñez, 2013, p. 105).

Por su parte, y en línea con esta aproximación a la escuela como comunidad política de pertenencia y reconocimiento, Larrondo (2017) ha resaltado lo importancia del proceso de «escolarización de la política» que tiene lugar en las escuelas. Según sostiene la autora, a través de una serie de dispositivos las instituciones escolares habilitan (u obstaculizan) la participación y/o emergencia de lo político en su interior, lo que incluye cierto control de la conflictividad (2017, p. 126). Esta forma de administración de la participación de los jóvenes supone entonces la distinción entre niveles o grados de politización de las prácticas. Así, sería posible distinguir entre éstos en función de la capacidad de articulación del interés colectivo -lo que supone la conformación de una identidad colectiva- y de la conflictividad entre las posiciones sociales de los actores (Larrondo, 2017, p. 118). La idea de politización como posibilidad nos exige, entre otras cosas, prestar atención a los alcances y las limitaciones en la inclusión de la voz estudiantil, junto con las condiciones sociales en las que se produce la escucha, en los distintos niveles de toma de decisiones que hacen al gobierno escolar.

Como hemos podido apreciar hasta aquí, la relación entre educación ciudadana, participación y política ha sido profusamente estudiada en Argentina. Sin embargo, hay ciertos aspectos de la cuestión que permanecen aún hoy descuidados, como es el caso de algunos de los dispositivos que efectivamente se ponen en juego en el cotidiano escolar. En este sentido, cabe recuperar aquí una serie de debates que ofrecen algunas alternativas al momento de registrar y contrastar la clase de diferencias y desigualdades que se manifiestan y tienen directa vinculación 
con las experiencias formativas de los y las jóvenes. Me refiero con ello puntualmente al estudio de la discusión de temas controvertidos en las escuelas.

Según ha mostrado Laura Quaynor, en aquellos países que atraviesan períodos de postconflicto, como es el caso de guerras civiles o dictaduras militares, es posible identificar cierta tendencia a evitar temas controvertidos en las aulas, en la medida en que ello supondría de algún modo enfrentar y avivar las tensiones acumuladas (Quaynor, 2011, p. 45). Referir a aspectos globales de la ciudadanía en desmedro de los nacionales (a nivel curricular), así como también evitar el contacto interétnico o divulgar información personal (como estrategia de los actores), aparecen como modos concretos de garantizar esa evitación.

Por otra parte, Quaynor presenta como más o menos saldada la cuestión de si la discusión de temas controvertidos puede conducir en algún caso a prácticas y modos de vinculación no democráticos. Para ello refiere al trabajo de Mapiasse (2007) en Indonesia como un modo de ilustrar esta relación negativa, que puede deberse al hecho de que en aquellas situaciones en que los maestros plantean problemas controvertidos, actúan como los recursos últimos de las opiniones finales frente a sus estudiantes. Detrás de todo esto está el supuesto de que la discusión abierta de temas controvertidos requiere no solamente de la disposición del profesor para renunciar al control intelectual de aquello que sucede en el aula, sino también de la disposición de los estudiantes a participar en la peligrosa empresa de hablar sobre las diferencias (Quaynor, 2011, p. 45). Vale destacar que esto implica cierta prescripción normativa amparada en un tipo de ciudadanía democrática en la cual la legitimidad ha de ser discutida y discutible, a sabiendas de que lo que está en juego es la construcción de un vínculo democrático entre individuos desiguales (Dubet, 2003).

Al prestar atención a la dimensión política de las prácticas y las experiencias escolares, resulta sencillo reconocer una gran variedad de referencias a temas controvertidos, tanto en las aulas como en los pasillos de las escuelas en Argentina. A lo largo de mi trabajo de campo he podido registrar paredes repletas de afiches que registran las reflexiones y opiniones de los estudiantes, según las efemérides que los docentes introducen en sus planificaciones, y que recuerdan desde acontecimientos como la última dictadura militar o la «noche de los lápices» hasta supuestos hechos de corrupción cometidos por dirigentes políticos contemporáneos. Asimismo, «discutir» aparece como un elemento articulador de cierta clase de experiencias y sentidos que están en juego en las aulas y que cobran especial importancia durante la clase de ciudadanía ${ }^{2}$. Es un dispositivo en el sentido pedagógico pero, más importante aún, es la expresión visible de las tensiones y conflictos que los propios actores dirimen, desnudando la dimensión relacional y coproducida de la dinámica misma de la clase. El «discutir», en tanto práctica habitual que se desenvuelve durante el desarrollo

\footnotetext{
${ }^{2}$ Con esto me refiero a los espacios curriculares específicamente destinados a abordar cuestiones relativas a la educación ciudadana. En el caso de la Provincia de Córdoba, estas asignaturas comprenden "Ciudad $\mathbf{6}$ ńa y Participación" (10 y 2 Año del Ciclo Básico) y "Ciudadanía y Política" (6º año del Ciclo de Orientación). Los estudiantes suelen referirse a ambas como la «clase de ciudadanía», con lo cual termina por configurarse como un categoría nativa.
}

Praxis educativa UNLPam, Vol. 25, No 3, septiembre-diciembre 2021, E - ISSN 2313-934X, pp. 1 - 17. 
de la clase de ciudadanía, aparece como un elemento nodal que permite identificar las diferentes variantes que se inscriben en un tipo particular de formación ciudadana. En este sentido, considero que el análisis de la «discusión» resulta especialmente útil en la medida en que se ubica precisamente en medio de la tensión entre el estudio de la agencia política de los colectivos sociales y de la formación histórico social de aquellas instituciones que conforman la organización política de una sociedad, distribuyendo derechos y asignando responsabilidades de manera más o menos justa. De este modo, permite avanzar en un tipo de análisis que entiende a la formación ciudadana en clave de estructuras, relaciones y procesos.

\section{Metodología}

Los resultados que aquí se ponen en consideración forman parte de un estudio de casos realizado en tres escuelas secundarias de la ciudad de Córdoba (Argentina). Todas ellas atravesaron procesos de «tomas» durante los conflictos que tuvieron lugar en 2010, en el marco de los debates por la sanción de la nueva Ley de Educación Provincial No 9.870, actualmente vigente. Si bien esto puede sugerir un nivel de organización y participación estudiantil destacado, al reconstruir la historia reciente de cada escuela es inevitable encontrarse con trayectorias sumamente heterogéneas. El primer caso ${ }^{3}$ que denomino Escuela Superior de Comercio Guillermo Bustos se caracteriza por atender a más de 1200 estudiantes provenientes de sectores de nivel socioeconómico heterogéneo (en su gran mayoría de niveles medios y medios-bajos). Se trata de una escuela de gestión pública muy tradicional de la ciudad, reconocida por formar parte del selecto grupo de las escuelas "palacio", cuyas virtudes arquitectónicas devinieron recientemente en foco de conflicto, en un contexto marcado por millonarios emprendimientos inmobiliarios a lo largo y ancho de la ciudad. El segundo caso, denominado Instituto Provincial de Educación Media No 67 Salvador Allende (o simplemente Allende, como lo llaman estudiantes y docentes), hace lugar a cerca de 700 alumnos, provenientes en su gran mayoría de sectores populares. Esta escuela, también de gestión pública, está emplazada en cercanías de uno de los barrios más grandes y populares de la ciudad, por fuera del anillo de circunvalación. Finalmente, el tercer caso elegido será referido como Colegio Nuestra Señora de la Concepción -o simplemente Concepción-. Pese a lo

\footnotetext{
${ }^{3}$ Cabe aclarar que, tal como se acordó con estudiantes, docentes y directivos, se ha garantizado el anonimato de todos aquellos que participaron directa o indirectamente en esta investigación. Es por ello que han modificado no solo los nombres de los protagonistas, sino además los de cada una de las escuelas seleccionadas. He intentado mantener, sin embargo, algunas de las características que aparecen involucradas en los modos de nombrar. Así, procuré respetar los modos en que ellos mismos construyen sentidos a través de determinadas estructuras de significantes, entendiendo que esa es una clave también de los modos de relacionarse e interactuar. Podrá notarse, por ejemplo, la sinécdoque que opera detrás del modo habitual que tienen los estudiantes de referirse a "Rocco", "Mari" o "López". Si bien estos son los nombres o apellidos de aquellos docentes que están a cargo de la asignatura Ciudadanía y Política en cada una de las escuelas, sus figuras resultan intercambiables respecto a aquello que llaman "la clase de ciudadanía" o simplemente "ciudadanía".
}

Praxis educativa UNLPam, Vol. 25, No 3, septiembre-diciembre 2021, E - ISSN 2313-934X, pp. 1 - 17. 
que su nombre sugiere, se trata de un colegio laico de gestión privada reconocido por contar con una planta docente que en gran parte se inscribe en las llamadas pedagogías críticas. Asisten allí alrededor de 350 estudiantes, provenientes en su mayoría de sectores medios y sobre todo mediosaltos.

En lo que refiere al diseño metodológico se optó por un enfoque etnográfico, en correspondencia con un extenso trabajo de campo que incluyó la observación y registro de las «clases de ciudadanía» a lo largo de todo el ciclo lectivo de 2016, junto con la realización en simultáneo de 43 entrevistas y grupos de discusión, con el objeto de recuperar el punto de vista de los estudiantes, esta vez, fuera del alcance y la mirada de los docentes. El carácter abierto de la estrategia inicial, que tomó como base las observaciones de las dinámicas durante el desarrollo de las asignaturas específicamente destinadas a la formación ciudadana, me llevó luego a recorrer distintas sendas de indagación junto con los actores, en función del despliegue que cada caso propuso. Así es como terminé acompañando a estudiantes y docentes a talleres, usentadas», cortes de calle, reuniones del Centro de Estudiantes y reuniones de docentes y padres, entre otros espacios y momentos de encuentro y discusión.

Acuerdo con Levinson respecto a la pertinencia y potencia que acompaña a la descripción etnográfica acerca de la interacción entre las prácticas y los discursos educativos en el terreno de la ciudadanía informal (2011, p. 293). En ese sentido, estoy convencido de que una perspectiva antropológica de esta naturaleza puede hacer una contribución significativa. Un estudio comparativo, aún desde un enfoque etnográfico (que preste atención a la riqueza y a la diversidad de prácticas y sentidos desde el más pequeño de los detalles), permite identificar una estructura similar en las clases de ciudadanía, solapando por momentos la gran heterogeneidad que caracteriza a las trayectorias de los docentes y estudiantes que forman parte de las tres comunidades educativas elegidas para este estudio. Eso recuerda el sentido y la apuesta inicial de estudiar la ciudadanía en las escuelas, antes que el hallazgo de una regularidad o recurrencia social. La configuración de experiencias y modos de habitar, en todo caso, explican cierto devenir abierto y diverso. En este sentido, no se trata de entender a la formación ciudadana en un sentido unívoco, mucho menos desde una perspectiva sistémica o estructural-funcionalista. Es más bien la expresión de un modo de abordar y comprender la clase de dinámicas y tensiones que están en juego en un escenario y un momento determinado, prestando atención especialmente a los modos en que se expresa la política en las escuelas secundarias. La estrategia aquí asumida responde justamente a la necesidad de emprender abordajes etnográficos, desde una mirada puesta sobre las prácticas políticas, con el objeto de profundizar la reflexividad en medio de las intersecciones que el etnógrafo habita.

\section{Resultados}

Una de las primeras cuestiones que es necesario destacar al momento de emprender el análisis de las discusiones que tienen lugar durante el desarrollo de las clases de ciudadanía es el carácter ubicuo y estructurante que ellas mismas denotan. En otras palabras, la «discusión» aparece 
como un dispositivo sumamente recurrente que vehiculiza otras prácticas, dándole forma a cierta clase de tensiones que cada comunidad educativa acumula en su propio devenir, ofreciendo al mismo tiempo algunas alternativas en lo que respecta a prácticas políticas o formas de ejercicio de la ciudadanía. Según he podido observar a lo largo de mi trabajo de campo, la «clase de ciudadanía» configura un tiempo-espacio escolar en el que la discusión e intercambio de puntos de vista acerca de distintas problemáticas ocupa un lugar central, condensando gran parte de las disposiciones y las expectativas que estudiantes y docentes ponen en juego allí cada semana. Esta primera aproximación a la «discusión» como un dispositivo más -en el sentido foucaultiano- que configura las dinámicas escolares, nos orienta en la búsqueda de una función estratégica concreta, así como también en la identificación del juego de relaciones de poder y de saber en el que se inscribe (Agamben, 2011).

Sin embargo, una mirada enfocada exclusivamente en la discusión como parte de las "técnicas" de socialización que constituyen el trabajo educativo no resulta suficiente en el marco del llamado declive del programa institucional (Dubet, 2010). Bajo determinadas circunstancias, que por cierto deben ser precisadas, la discusión se presenta como una escenificación dramatúrgica. Esto supone una crisis del flujo diario de las interacciones sociales, la cual puede en ciertas ocasiones llegar a expresar la fuerza de un drama social, entendido este como una unidad de procesos armónicos o no armónicos que surge en el marco de determinadas situaciones de conflicto (Turner, 2002). Si destaco la potencia de ensayar una antropología de la performance ritual es debido al hecho de que allí es posible articular estructura y acción, identificando los intersticios en los que se torna visible la esfera de la liminalidad, en tanto momento de desorganización de las formas sociales y, por lo tanto, de gran creatividad.

Al prestar atención al lugar que ocupa la discusión desde el punto de vista de los docentes, es evidente que este dispositivo cobra especial importancia desde el inicio mismo de la clase de ciudadanía. Este es el caso de las clases de Rocco, docente del Colegio Concepción, quien hace algunos años decidió incorporar en su propia planificación un segmento inicial de la clase en el que debate junto a sus estudiantes acerca de temas políticos de actualidad nacional e internacional, que por lo general han gozado de una amplia difusión mediática en los días previos. Este umbral de la clase suele representarse de un modo sumamente estructurado y es el propio docente quien decide qué temas pueden discutirse y cuándo es el momento adecuado para dar por cerrada la discusión. Mari, docente del Instituto Allende, por su parte, suele hacer lugar a un tramo inicial de su clase en el que se van alternando discusiones acerca de cuestiones personales y problemas públicos sin una estructura demasiado rígida. Esto, mientras preparan los equipos de audio y video en los que se proyectará el material audiovisual del día. Así, en este primer tramo, pueden conversar sobre cómo avanza el embarazo de una de las estudiantes, intercambiar opiniones acerca del aborto o evaluar las implicancias de una polémica declaración del presidente de Estados Unidos. En el caso de López, docente de la Escuela Gobernador Bustos, se puede apreciar el gran esfuerzo que demuestra a lo largo de toda la clase con el objeto de iniciar algún tipo de debate. Sin embargo, y a diferencia de los otros dos casos, suele encontrarse con una férrea resistencia por parte de los y las estudiantes. 
Estas dificultades se manifiestan aún en aquellas circunstancias en que recurre a exclamaciones abiertamente polémicas (a veces incluso con un claro sesgo sexista), como un modo de apelar a la indignación de su audiencia y motivar una reacción que alimente el intercambio. La discusión frustrada da cuenta de un fracaso sistemático, que en el caso de la Escuela Bustos se explica en gran parte por las referencias y argumentos que el docente esgrime en torno a cuestiones de género y sexualidad. La posición de rechazo de las estudiantes mujeres impone un límite claro a partir del cual la dramatización se transforma en un simple monólogo, mientras un grupo de estudiantes copian lo que el docente escribe en la pizarra. En tanto dispositivo, la discusión frustrada expresa una relación de fuerzas en la que se impone la evitación mutua. Mientras el docente escribe dos a tres pizarras completas por clase, los estudiantes modelan un repertorio propio en el que escaparse del aula, jugar a las cartas o escuchar música en el celular ocupan un lugar central.

Por otra parte, al retomar puntualmente aquellos casos en que la discusión se muestra como relativamente exitosa -desde el punto de vista de los objetivos y las estrategias implicadas en el propio dispositivo-, podemos observar algunas diferencias más que es preciso analizar. Este segundo nivel de diferenciación entre los casos estudiados exige hacer foco en el punto de vista de los estudiantes y en las condiciones en las cuales los jóvenes se enganchan con la dinámica de la discusión y el horizonte que ella habilita. Claro que este relativo éxito no implica homogeneidad alguna. En este sentido, el reconocimiento por parte de los estudiantes, que en general valoran el espacio-tiempo de la discusión que les ofrece Rocco (a veces incluso demandan "discutir más"), se ve empañado por el hecho de que algunos consideran que estos intercambios "no conducen a nada". La ausencia de conclusiones y la sensación de que no pueden "aplicar" lo conversado en ningún ámbito en particular confluyen en la representación de que algunos de ellos solo discuten "para perder tiempo". En el caso del Instituto Allende, por otra parte, el visible éxito de las discusiones durante la clase de ciudadanía, se ve contrarrestado en situaciones de entrevista, lejos de la mirada de la docente. Allí, algunos estudiantes manifiestan rechazo frente a lo que entienden es una clara y sostenida intencionalidad por parte de la docente durante las discusiones. En este sentido, cuestionan que Mari quiere "Ilenarles la cabeza", a pesar de que sostenga lo contrario. Una discusión sobre cuestiones político-partidarias entre un estudiante y la docente, en la cual esta última aprovecha de algún modo una situación de ventaja en el manejo de la información, suele sedimentar en posiciones de rechazo solapado por parte de algunos estudiantes frente a la dinámica propuesta. Con estas palabras representaban la situación dos estudiantes del Allende:

J...: Ella dice que no nos quiere llenar la cabeza con algo que opina ella, pero para mí sí.

P...: Sí, porque nos quiere meter opiniones de ella a nosotros.

J...: Ella dice, "todos son libres de opinar". El otro día las chicas le dijeron a L... lo de Macri, algo de Macri, que no lo dejaban ser buen presidente. Lo criticaban ahí nomás los primeros días. Y ella empezó, "Si no ven que les aparece plata por todos lados". Empezó a dar opiniones de ella contradiciendo las de la otra, pero como le quería meter en la cabeza una idea de ella. Y eso es lo que para mí está mal, no sé. Yo por eso no hablo en la clase de ella. 
Cabe destacar, sin embargo, que esta delgada línea por la que los docentes suelen caminar goza de un amplio margen de interpretación por parte de los jóvenes. Así, pude observar también en una ocasión a un grupo importante de estudiantes que se manifestaron públicamente durante la clase en agradecimiento a la docente por "enseñarles de política" en el marco de esas mismas discusiones. En el caso del Colegio Concepción, por su parte, cabe destacar el hecho de que los estudiantes rechazan de plano que los docentes intenten convencerlos o imponer algún tipo de mirada desde el punto de vista político-ideológico. Antes bien, lo que intentan es "abrirles la cabeza". Así lo manifiestan dos de los estudiantes:

M...: siempre nos parece bien que el profe venga y te diga: "Bueno, yo tengo esta...[idea]". Bueno, no que venga y sea como una confesión de: "Yo pienso esto", sino de que siempre esté abierto a discutir sobre eso. Y creo que la mayoría de los profes piensa lo mismo, que es una forma de interpretar que es diferente a las otras formas de interpretar.

H...: Ahora estamos viendo al pelado Lenin. No sé, eso sí me encanta, porque es algo que está bueno saberlo porque son corrientes que a mí me abrieron la cabeza un poco y que no están enseñadas bien en otros colegios.

Finalmente, aunque no menos importante, cabe resaltar una serie de diferencias entre los casos del Colegio Concepción y el Instituto Allende, que permiten observar ciertos clivajes en lo que respecta a los horizontes de liminalidad y creatividad. Me refiero con ello a la importancia de identificar qué discusiones ganan terreno como escenificaciones dramatúrgicas que pueden conducir potencialmente a situaciones de conflicto (que como ya dijimos expresan a su vez dramas sociales). Para ello me detendré en dos momentos particulares que forman parte de las unidades procesuales en las cuales la discusión ocupa un lugar central y organizador.

Por un lado, respecto del caso del Instituto Allende, cabe destacar el hecho de que habitualmente las discusiones giran en torno a injusticias vividas en primera persona. Situaciones de violencia institucional o delincuencial, pobreza, corrupción, desocupación o acceso a la vivienda, entre otros, suelen ser los disparadores de los intercambios entre posiciones y opiniones. En mis observaciones pude incluso registrar el modo en que dos de estas discusiones dispararon reclamos por las condiciones edilicias y los robos sufridos por la escuela, lo que llevó a una sentada y un corte de calle, en tanto modalidades de acción directa emprendidas por los propios estudiantes en colaboración con la docente. En el caso del Colegio Concepción, por otra parte, resalta el hecho de que las injusticias que son abordadas en las discusiones son sufridas por otros. Esto abre la posibilidad de un cambio de escala en las problemáticas debatidas, habilitando discusiones a nivel nacional e internacional, que se complementan con cierto nivel de teorización social y política. Un ejemplo que puede ilustrar este contraste resulta de una discusión en torno a la problemática habitacional y el derecho a la vivienda. Mientras los estudiantes del Instituto Allende discutían acerca de los programas de financiamiento del gobierno provincial para el acceso a terrenos, y las condiciones impuestas que los dejaban afuera por no contar con los ingresos suficientes, los estudiantes del Concepción analizaban el caso de la toma de tierras en el Parque Indoamericano de la Ciudad Autónoma de Buenos Aires en 2010, desde la mirada de Thomas Hobbes, John Locke y 
Alexis de Tocqueville. Esta diferencia termina de comprenderse si se tiene en cuenta que la única situación problemática a la que condujo una de las discusiones durante la clase de ciudadanía del Colegio Concepción, estuvo ligada al cuestionamiento del diseño de los contenidos del programa de la asignatura. Este momento, ciertamente liminal, condujo a la reflexión conjunta del docente y sus estudiantes acerca de la necesidad de reorientar el curriculum hacia una propuesta más bien práctica, en torno a la realización de diversas actividades y reuniones junto con otros colegios y centros de estudiantes de la ciudad. Si bien esto provocó el conflicto con la Directora, quien no estuvo de acuerdo con que Rocco modificara el programa de la materia cediendo a los estudiantes la autoridad pedagógica, el problema no pasó a mayores.

El segundo momento que creo importante recuperar, y que forma parte también de la unidad procesual centrada en la discusión como dispositivo y drama social, es aquel que se corresponde con los modos en que ésta logra reconfigurar el vínculo entre los estudiantes y sus familias. Según se desprende de los testimonios de los propios jóvenes, las discusiones y aprendizajes que se enmarcan en las clases de ciudadanía tienen una clara repercusión al interior del seno familiar. En el caso de los estudiantes de Instituto Allende, he podido identificar las marcas de una suerte de alfabetización política, que se manifiesta a modo de un capital que los propios estudiantes acumulan en sus experiencias escolares y reinvierten en la vida cotidiana familiar. Los propios estudiantes dan cuenta de esto al referir a escenas de discusiones familiares en las que logran sobreponerse ante la autoridad paterna, a partir de conocimientos y habilidades generadas en las clases de Mari.

J...: Sí. Yo la otra vez me largué a pelear con mi mamá por eso, porque mi mamá me decía, "Vos no sabés nada". Le digo, "¡Cómo que no voy a saber si estudio esto!", "¡Vos no sabes nada!", le digo. Y un momento le dije, "Sos una ignorante". Le dije así, le dije vos no sabés nada. Pero porque me decía cosas que le digo, "Mami, yo eso lo estoy estudiando y lo estoy viendo". Y encima, justamente, lo bueno que tenemos que el profe de Historia también nos da muy mucha política, pero él es radical y la Mari es peronista. Entonces es como que estamos de los dos, como que nos da este y nos da este.

En lo que respecta al caso del Colegio Concepción, en cambio, los estudiantes refieren a estrategias de evitación de las discusiones políticas que ocasionalmente surgen en el seno familiar. Esto implica la acumulación de ciertas tensiones que tienen su origen en una clara oposición, no solo entre lo que la escuela y las familias enseñan a sus hijos, sino además entre las orientaciones y posiciones políticas que caracterizan a docentes y padres. En este sentido, algunos de los jóvenes reconocen que en cierto modo hacen de resorte, amortiguando los potenciales conflictos que esto pudiera ocasionar. No contar demasiado acerca de lo que aprenden en la escuela es una de las tácticas a las que recurren. En medio de todo ese proceso, mucho de ellos manifiestan una clara toma de conciencia de la arbitrariedad que supone la autoridad paterna. Para ilustrar esto, reproduzco un pasaje de una de las entrevistas con estudiantes del Colegio Concepción:

F...: No se le puede decir nada, mi viejo es una persona que como padre es buena pero como persona no tanto. Digo, bueno, evito hablar de esos temas con él y me centro en lo bueno 
que puedo rescatar. [...] Si. Yo soy diferente en el sentido que nunca me nutrí por las ideas que me dio... por muchas ideas, o sea, las ideas básicas o de eje que me dio mi familia. Sino creo que hubiera sido otro tipo de persona. Y que más me llevó a confiar en el colegio en esas ideas y por lo tanto ver y ver que se puede criticar tanto a mi viejo. Por ejemplo, una cosa, mi hermana que es mayor que yo, tiene 21 años, tiene a la palabra de mi viejo como palabra santa. Indiscutible. Y yo digo que no, hay miles de cosas que se le pueden criticar a él como a cualquier otra persona. Pero si vos le decís a mi viejo que eso está mal, dice, "Cómo me vas a decir eso si soy tu padre". Entonces es como una falacia, porque soy yo no me podés cuestionar. Por dentro te agarra rabia, pero no podés hacer nada.

Como se puede apreciar, discutir y no discutir aparecen como facetas de un mismo proceso en el cual los y las jóvenes van tejiendo los alcances y los límites de sus propias experiencias escolares, mostrando modos específicos de configuración y politización de su formación ciudadana. Así, la escuela, la clase de ciudadanía y la familia aparecen como experiencias individuales y colectivas entrecruzadas y articuladas. Si hay un carácter activo, liminal y creativo en estos procesos, su propio desenvolvimiento muestra diferencias y desigualdades muy claras, abriendo diversos horizontes de lo posible.

\section{Conclusiones}

Hasta aquí hemos intentado profundizar el análisis de las prácticas y representaciones de los jóvenes, en línea con una perspectiva tal que sea capaz de reconocer la densidad y la riqueza de las experiencias y los modos de habitar las escuelas. Así, los datos recogidos y coproducidos permiten hacer visibles las relaciones que mantienen docentes y estudiantes como parte de un vínculo político que solamente resulta comprensible en la textura misma de la vida cotidiana escolar. A lo largo de estas páginas he intentado mostrar cómo la «discusión», en tanto dispositivo y escenificación observada y observable en el marco de las clases de ciudadanía, permite identificar y precisar cierta clase de diferencias y desigualdades que se expresan en los modos de construcción de ciudadanía. De este modo, la discusión exitosa se diferencia de la frustrada, en la medida en que los jóvenes encuentran y reconocen (o no) en sus docentes una oportunidad para la construcción de saberes a partir del intercambio de opiniones y puntos de vista sobre las problemáticas actuales. El interés demostrado por los docentes, en lo que respecta a orientar estas discusiones ponderando la dimensión política de las relaciones sociales, supone cierto riesgo de que esos esfuerzos sean interpretados como parte de una estrategia de manipulación o adoctrinamiento desde el punto de vista político-ideológico. «Llenar» o «abrir» la cabeza aparecen como las principales categorías nativas a partir de las cuales se identifican algunos de los sentidos estratégicos involucrados. En cambio, si hay algo en lo que coinciden todos los actores, es en el hecho de que la clase de ciudadanía está (o debería estar) orientada fundamentalmente a la práctica. Esto da cuenta de un tipo de sedimentación del horizonte estratégico de los dispositivos escolares, en el cual la discusión ocupa un lugar muy importante. 
Finalmente, hemos analizado dos momentos liminales en los cuales se pueden apreciar distintas formas de desorganización y creatividad, bajo situaciones de conflicto que las escuelas en general, y las clases de ciudadanía en particular, ayudan a modelar. La distancia variable respecto a las problemáticas discutidas, en el sentido de si se trata de injusticias vividas en primera o en tercera persona, junto con los niveles de teorización alentados por los docentes, permite comprender las razones de que la educación ciudadana resulte (o no) en una experiencia práctica y activa de aprendizaje y conocimiento. Protestar en reclamo por la mejora de las condiciones edilicias y tener injerencia en el diseño curricular, aparecen como dos elementos muy distintos en medio de un abanico de posibles que requiere ser ampliado y completado, pero que ofrece igualmente algunas coordenadas para pensar qué clase de derechos caracterizan a cada sector social. El segundo momento liminal, relativo a las transformaciones que tienen lugar en el marco de los vínculos entre padres/madres e hijos/hijas, da cuenta de los distintos modos en que cada sector tramita sus propias tensiones y relaciones intergeneracionales. Lo que pudimos apreciar aquí es que mientras hay discusiones que se encadenan unas a otras, trascendiendo los límites temporales y espaciales que caracterizan a la clase de ciudadanía, otras se interrumpen abruptamente. Las estrategias que los propios jóvenes desarrollan, muestran los alcances y los límites de la escuela como escenario y vector de discusión y formación política. Esto no es otra cosa que una fotografía más de la tensa relación que mantienen familias y escuelas, y que sin dudas se encuentra también atravesada por condiciones socioeconómicas.

Si la «discusión» aparece como una categoría relevante de análisis, en el marco de las diferentes experiencias de formación ciudadana que tienen lugar en las escuelas secundarias, es por el hecho de que no se trata de una práctica esporádica o aislada. En cambio, su recurrencia se inscribe en las dinámicas escolares más cotidianas, mostrando las diferencias y desigualdades que allí se manifiestan. La discusión, como dispositivo y escenificación, permite reconstruir parte de la compleja trama a partir de la cual la escuela se configura como un escenario más de las disputas y las tensiones que caracterizan a la dimensión política de las relaciones sociales. 


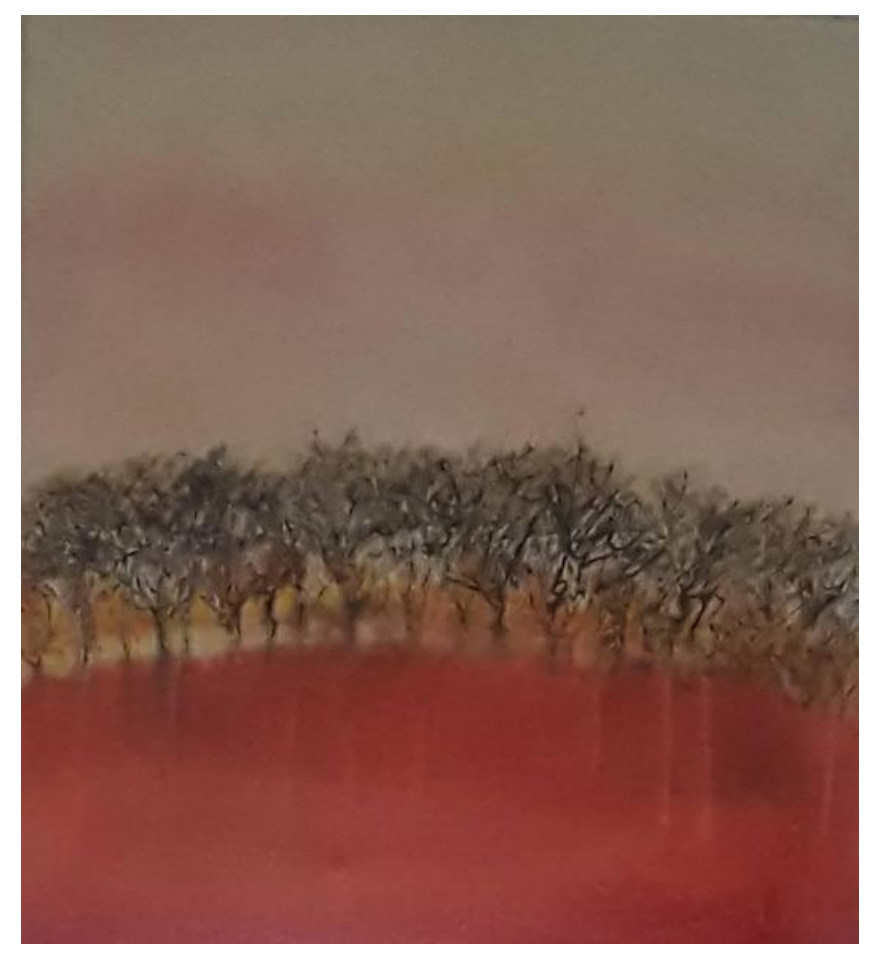

Montecito, técnica mixta. Adriana Chavarri

\section{Bibliografía}

Agamben, G. (2011). ¿Qué es un dispositivo? Sociológica, 249-264.

Agudo de Córsico, M. C. (1995). La Ley Federal 24.195 y la evaluación de la calidad de la educación. Serie Pedagógica, 155-168.

Alucín, S. (2018). Luces y sombras de la política en la escuela secundaria:breve historización de la educación ciudadana. Revista SAAP, 45-70.

Ascolani, A. (2000). LA "Revolución Libertadora": educación y ciudadanía restringida (Argentina, 1955-1958). 23a Reunión Anual. Caxambu: ANPED.

Dubet, F. (2003). Mutaciones cruzadas: la ciudadania y la escuela. En J. Benedicto, \& M. L. Morán, Aprendiendo a ser ciudadanos. Experiencias sociales y construcción de la ciudadanía entre los jóvenes. Madrid: Injuve.

Dubet, F. (2010). Crisis de la trasmisión y declive de la institución. Política y sociedad, 47(2), 15-25.

Encabo, A. (2008). La enseñanza de la formación ciudadana por medio de las normas curriculares. En V. Batiuk, A 25 años de la democracia: las políticas para el área de Formación Ética y Ciudadana de la Educación Secundaria (págs. 35-55). Buenos Aires: Cippec.

Ferreyra, H. A., Romero Blanca, L. P., \& Vidales, S. N. (2014). La formación ciudadana en la el currículum escolar de la Provincia de Córdoba - Argentina. Revista Colombiana de Ciencias Sociales, 197-213.

Ferreyra, H. A., Scasso, M. G., \& Patiño Mayer, M. (2013). La educación secundaria en Argentina: una mirada estadística de las trayectorias escolares. Magistro, 25-50.

Hernández, A. (2019). Ciudadanía, democracia y participación: un estudio de casos en escuelas secundarias de la Ciudad de Córdoba (Argentina). Polifonías, 17-53. 
Kriger, M. (2016). La tercer invención de la juventud: dinámicas de la politización juvenil en tiempos de la reconstrucción del Estado-Nación (Argentina, 2002-2015). Buenos Aires: Grupo Editor Universitario.

Larrondo, M. (2017). Participación y escolarización de la política: Reflexiones sobre lo político en la escuela. Universitas(26), 109-134.

Levinson, B. (2011). Toward an anthropology of (democratic) citizenship education. En B. Levinson, \& P. Mica, A companion to the anthropology of education (págs. 279-298). Blackwell publishing.

Litichever, L. (2014). La Convivencia como modalidad de resolución de conflictos. I Encuentro Internacional de Educación. Tandil.

Litichever, L., \& Núñez, P. (2005). Acerca de lo justo, lo legal y lo legítimo. Cultura política en la escuela media. Última Década, 103-130.

Mapiasse, S. (2007). Influence of the democratic climate of classrooms on student civic learning in North Sulawesi, Indonesia. International Education Journal, 393-407.

Núñez, P. (2013). La política en la escuela. Buenos Aires: La crujía.

Núñez, P. (2014). La construcción de ciudadanía en la escuela secundaria: convivencia, regulación de la participación juvenil y nuevas dinámicas de la desigualdad. Revista de la escuela de ciencias de la educación, 35-52.

Núñez, P. (2019). La construcción de la ciudadanía: dinámicas de desigualdad en la experiencia escolar juvenil. Revista Estado y políticas públicas, 123-145.

Núñez, P., \& Litichever, L. (2015). Radiografías de la experiencia escolar. Buenos Aires: Grupo Editor Universitario.

Quaynor, L. (2011). Citizenship education in post-conflict contexts: a review of the literature. Education, citizenship and social justice, 33-57.

Schujman, G. (2008). Tensiones teóricas en la enseñanza de ciudadanía y derechos humanos. En V. Batiuk, $A$ 25 años de democracia: Las políticas para el área de Formación Ética y Ciudadana en la educación secundaria (pág. 142). Cippec.

Siede, I. (2007). La educación política : Ensayos sobre ética y ciudadanía en la escuela. Buenos Aires: Paidós.

Siede, I., \& Schujman, G. (2007). Ciudadanía para armar: Aportes para la formación ética y política. Aique: Buenos Aires.

Siede, I., Guglielmino, E., Alcain, J., Fernández, G., \& Guinao, D. (2015). Formación ética y ciudadana. Vicisitudes de la transformación curricular en la Patagonia Argentina. Folios, 51-68.

Siede, I., Guglielmino, M. E., Alcain, J., \& Fernández, G. (2016). Situación actual de la formación ética y ciudadana en las escuelas primarias del norte de Santa Cruz. Informe científico técnico UNPA.

Suárez, D. (2008). Rewriting citizenship? Civic education in Costa Rica and Argentina. Comparative Education, 485-503.

Turner, V. (2002). Antropología del Ritual. México D.F.: INAH-ENAH.

Turner, V. (2002). La antropología de la performance. En I. Geist, Antropología del Ritual: Víctor Turner (págs. 103-144). México D.F.: INAH-ENAH.

Vázquez, M. (2015). Juventudes, políticas públicas y participación. Buenos Aires: Grupo Editor Universitario. 
La "discusión" en las aulas. Un estudio sobre las experiencias de formación ciudadana en escuelas secundarias de Córdoba, Argentina | Andrés E. Hernández 